\title{
Optimized pinhole geometry for small-angle scattering
}

\section{András Wacha}

J. Appl. Cryst. (2015). 48, 1843-1848

\section{IUCr Journals \\ CRYSTALLOGRAPHY JOURNALS ONLINE}

Copyright (C) International Union of Crystallography

Author(s) of this paper may load this reprint on their own web site or institutional repository provided that this cover page is retained. Republication of this article or its storage in electronic databases other than as specified above is not permitted without prior permission in writing from the IUCr.

For further information see http://journals.iucr.org/services/authorrights.html 
JOURNAL OF

APPLIED

CRYSTALLOGRAPHY

ISSN 1600-5767

Received 1 August 2015

Accepted 7 October 2015

Edited by G. Kostorz, ETH Zurich, Switzerland

Keywords: small-angle X-ray scattering (SAXS); collimation; pinholes; small-angle scattering (SAS); parasitic scattering; signal-to-noise ratio.

Supporting information: this article has supporting information at journals.iucr.org/j

\section{Optimized pinhole geometry for small-angle scattering}

\author{
András Wacha* \\ Research Centre for Natural Sciences, Hungarian Academy of Sciences, Magyar tudósok körútja 2, Budapest, Hungary. \\ *Correspondence e-mail: wacha.andras@ttk.mta.hu
}

In order to make the most of the low photon flux in laboratory small-angle X-ray scattering instruments, the experimental geometry has to be chosen carefully, with special stress on the beam-shaping system. The optimum collimation scheme should enable accurate measurements over the desired range of the scattering variable, while yielding the highest flux and the lowest possible instrumental background. In order to identify the best setting, a phase-space optimization of the collimating scheme is carried out in the present work, including constraints on the beam size at the sample position and on the detector surface. The resulting formulae are implemented in a Python script with a graphical user interface, to aid the planning, construction and daily operation of pinhole small-angle scattering cameras.

\section{Introduction}

Collimation is arguably the most important aspect of a smallangle scattering (SAS) instrument, as it is responsible for shaping the incident radiation by confining it in the transverse plane and limiting its divergence.

Several designs have been developed for the same task, including the Kratky-type block collimation (Kratky \& Stabinger, 1984; Fritz \& Bergmann, 2006; Bóta, 2013), the Huxley-Holmes design (Huxley et al., 1965; Huxley \& Brown, 1967; Zemb et al., 2003a,b) and the Bonse-Hart camera for ultra-small-angle scattering (Lambard \& Zemb, 1991; Rehm et al., 2013). In the widely used pinhole camera (e.g. Hendricks, 1978; Haubold et al., 1989; Narayanan et al., 2001; Jakob et al., 2003; Knaapila et al., 2009; Kirby et al., 2013) the beam is cut by two or more apertures made from absorbing materials.

The simplest apertures are circular pinholes. Variable-size slit systems represent another, more sophisticated alternative, where two pairs of independently movable blades placed just after each other limit the beam horizontally and vertically, resulting in a rectangular beam cross section.

The frequently used three-aperture scheme is shown in Fig. 1. Radiation enters on the left through the entrance aperture, placed as near as possible to the source. Together with the second, beam-defining aperture they limit the transverse size and maximum divergence. The third aperture is the anti-scatter slit or pinhole, because its purpose is to cut away most of the parasitic scattering which originates from the collimating elements upstream. Using scatterless pinholes and slits, however, can render the last aperture obsolete.

The distances between the various elements of the setup measured along the optical axis are denoted by $l$. Two different measures can be defined for the maximal transverse extent of the beam: in one the parasitic scattering transmitted through 
aperture \#3 is neglected ( $r^{\prime}$ in Fig. 1), while in the other (denoted by $r$ ) it is accounted for.

The first mathematical study on collimation in small-angle scattering measurements was by Bolduan \& Bear (1949). A sealed X-ray tube with a three-aperture collimating system was considered and the configuration for fastest camera speed was derived, given $r_{\text {det }}, r_{\text {det }}^{\prime}, r_{1}$ and the full camera length, $l_{1}+l_{2}+l_{3}$.

The case study of Wignall et al. (1990) underlines the importance of the third aperture. Installing an anti-scatter pinhole in a small-angle X-ray scattering (SAXS) apparatus (Schelten \& Hendricks, 1975; Hendricks, 1978) reduced the instrumental background by more than one order of magnitude.

Another article reports the design of a SAXS camera with two-pinhole collimation (Bu et al., 1998). The absence of the third aperture results in a considerable parasitic scattering, which the authors reduce by other means, e.g. increasing the thickness of the sample.

Pedersen (2004) carried out a phase-space analysis in order to minimize the background in a commercial SAXS apparatus. Flux maximization was done using a Monte Carlo approach based on a plausible model for the raw beam profile.

In this work a similar phase-space analysis to that of Pedersen is carried out for both background and flux optimization. The proof-of-concept instrument is a SAXS apparatus (Wacha et al., 2014) with circular apertures; the results presented here are nevertheless believed to be valid for neutron instruments as well and also applicable with little effort in cameras equipped with adjustable slits.

\section{Collimation geometry}

The fundamental parameters of the three-pinhole collimation scheme are the sizes $\left(r_{1,2,3}\right)$ of the apertures and the spacings $\left(l_{1}\right.$ and $l_{2}$ ) between them. Owing to its special role, the size of pinhole \#3 depends on the other four parameters:

$$
r_{3}=r_{1} \frac{l_{2}}{l_{1}}+r_{2}\left(1+\frac{l_{2}}{l_{1}}\right)=r_{1} \lambda_{0}+r_{2}\left(1+\lambda_{0}\right) .
$$

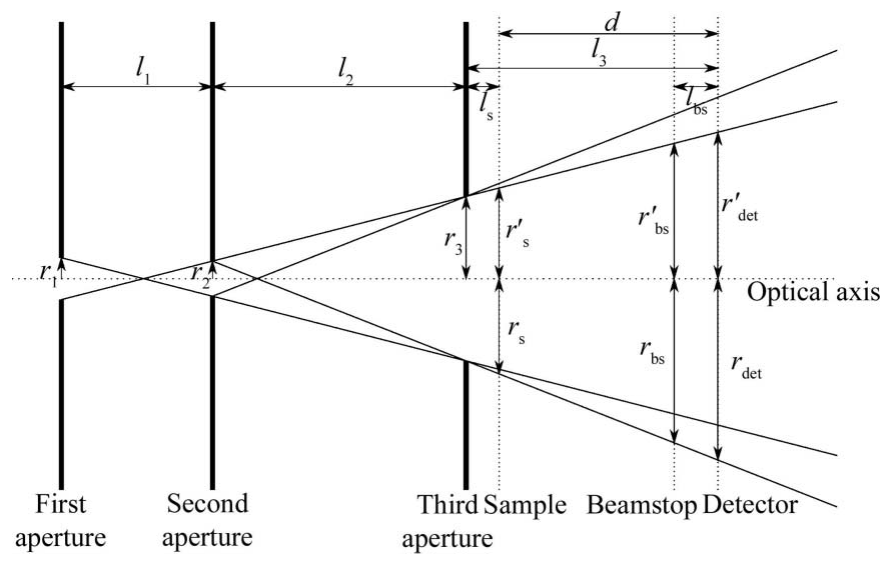

Figure 1

Schematic drawing of the three-pinhole collimation scheme.
The collimated beam has to meet two criteria: its cross section should not exceed that of the sample and the beamstop at the respective positions. The latter limitation is imposed by the smallest desired value of the scattering variable $(q=$ $4 \pi \sin \theta / \lambda, 2 \theta$ being the scattering angle and $\lambda$ the wavelength).

As parasitic scattering is not expected to make a measurable contribution to sample scattering, the relevant quantity for the beam size at the sample is $r_{\mathrm{s}}^{\prime}$. On the other hand, the beamstop must be large enough to cover it on the detector; therefore the correct measure for the beamstop size is $r_{\mathrm{bs}}$. From similar considerations to equation (1) we find

$$
\begin{aligned}
r_{\mathrm{s}}^{\prime} & \geq r_{1} \frac{l_{2}+l_{\mathrm{s}}}{l_{1}}+r_{2}\left(1+\frac{l_{2}+l_{\mathrm{s}}}{l_{1}}\right)=r_{1} \lambda_{1}+r_{2}\left(1+\lambda_{1}\right), \\
r_{\mathrm{bs}} & \geq r_{2} \frac{l_{3}-l_{\mathrm{bs}}}{l_{2}}+r_{3}\left(1+\frac{l_{3}-l_{\mathrm{bs}}}{l_{2}}\right)=r_{2} \lambda_{2}+r_{3}\left(1+\lambda_{2}\right) \\
& =r_{1} \lambda_{0}\left(1+\lambda_{2}\right)+r_{2}\left[\left(1+\lambda_{0}\right)\left(1+\lambda_{2}\right)+\lambda_{2}\right],
\end{aligned}
$$

where the auxiliary quantities $\lambda_{0}, \lambda_{1}$ and $\lambda_{2}$ have been introduced.

The distance between the third pinhole and the sample $\left(l_{\mathrm{s}}\right)$, as well as that between the detector and the beamstop $\left(l_{\mathrm{bs}}\right)$, is ideally very small, because a smaller beam size on the sample, as well as a larger beamstop, is almost always desirable. $l_{3}$ (or equivalently the sample-to-detector distance) can be adjusted in most pinhole instruments either continuously or in discrete steps.

The above two constraints ensure the low background of the instrument, leaving from the original four parameters only two independent. Assuming an analytical form of the primary beam intensity after the third pinhole, parameters for the maximum-flux geometry can be obtained.

If the flux of the X-ray source is homogeneous over the cross section of the first aperture, the transmitted intensity is

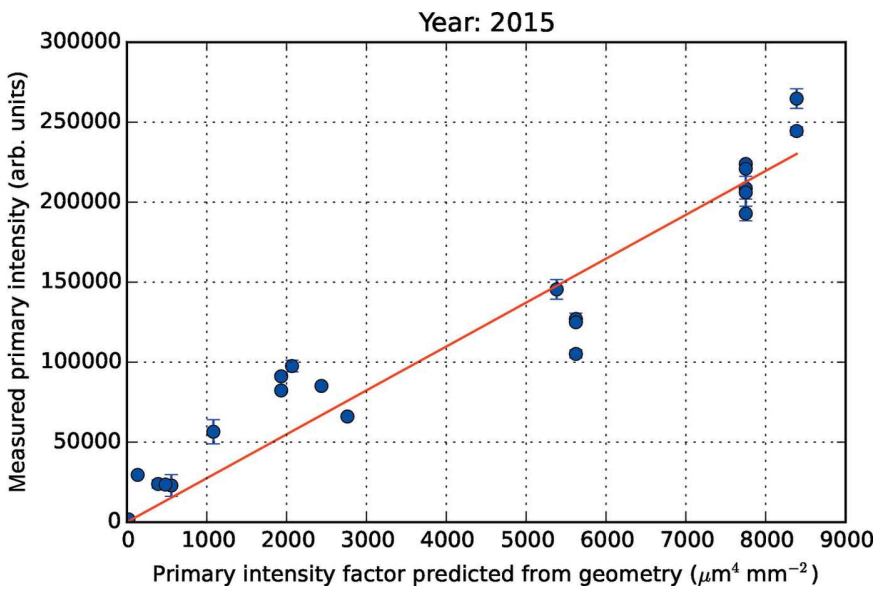

Figure 2

Measured versus predicted beam intensity. The data points correspond to different alignments of the CREDO instrument from January to July 2015. Measured intensities have been determined from the absolute intensity scaling factors of glassy carbon measurements from the same specimen. 
proportional to $r_{1}^{2} \pi$. Furthermore, if the directions of the rays passing through the entrance aperture follow a uniform distribution over the angular range defined by the first two pinholes, the first aperture is seen by the second one under approximately $r_{2}^{2} / 4 l_{1}^{2}$ solid angle. Since the third pinhole does not cut the main beam, the intensity at the sample is proportional to (Bu et al., 1998)

$$
\mathcal{I} \equiv \frac{\pi}{4} \frac{r_{1}^{2} r_{2}^{2}}{l_{1}^{2}}
$$

As noted previously, scattering of parasitic photons on the sample is neglected.

This simple approximation meets surprisingly well the results obtained on the test instrument. Fig. 2 shows a comparison of measured intensities to the corresponding values of $\mathcal{I}$. The slight deviations from linear behaviour can be ascribed to uncertainties of the determination of absolute intensity scaling factors and stability issues - or misalignment - in the X-ray source over several months.

Bolduan \& Bear (1949) made the disputable choice of using the intensity of the transmitted beam on the detector surface for characterizing the camera speed, which is proportional to $4 r_{1}^{2} /\left(l_{1}+l_{2}+l_{3}\right)^{2}$. The problem with this quantity is that it does not depend on $r_{2}$, and should be independent of $l_{3}$ if absorption in the flight path is negligible.

\section{Fixed pinhole distances}

In most SAS instruments the source is fixed; thus adjusting distances $l_{1}$ and $l_{2}$ would mean moving the remainder of the camera, which is usually not feasible. The spacings of the collimating elements are therefore fixed; only the choice of pinhole diameters is retained. Equations (2) and (3) translate to two constraints on $r_{2}$ in terms of $r_{1}$ :

$$
r_{2} \leq \frac{r_{\mathrm{s}}^{\prime}-r_{1} \lambda_{1}}{1+\lambda_{1}}
$$

for the sample size, and

$$
r_{2} \leq \frac{r_{\mathrm{bs}}-r_{1} \lambda_{0}\left(1+\lambda_{2}\right)}{\left(1+\lambda_{0}\right)\left(1+\lambda_{2}\right)+\lambda_{2}}
$$

for the beamstop size. $\left(r_{1}, r_{2}\right)$ pairs where the intensity is at its maximum can be found for both inequalities:

$$
\begin{gathered}
\left(r_{1, \mathrm{~s}}, r_{2, \mathrm{~s}}\right)=\left(\frac{r_{\mathrm{s}}^{\prime}}{2 \lambda_{1}}, \frac{r_{\mathrm{s}}^{\prime}}{2 \lambda_{1}+2}\right), \\
\left(r_{1, \mathrm{bs}}, r_{2, \mathrm{bs}}\right)=\left[\frac{r_{\mathrm{bs}}}{2 \lambda_{0}\left(1+\lambda_{1}\right)}, \frac{r_{\mathrm{bs}}}{2\left(1+\lambda_{0}\right)\left(1+\lambda_{2}\right)+\lambda_{2}}\right] .
\end{gathered}
$$

In the following the relation of the two constraints is analysed, i.e. if - and when - the fulfilment of one criterion implies the other one. Three possibilities exist ( $c f$. Fig. 3): the samplelimited, the beamstop-limited and an intermediate case.

In the first case the optimum of equation (2) satisfies equation (3). Substituting equation (7) into equation (6) yields an upper bound on the sample-to-detector distance, below which the sample size constraint is dominant:

$$
d \leq l_{2}\left[\frac{2 \lambda_{1}\left(1+\lambda_{1}\right)-\rho\left(\lambda_{0}+\lambda_{1}+2 \lambda_{0} \lambda_{1}\right)}{\rho\left(\lambda_{0}+2 \lambda_{1}+2 \lambda_{0} \lambda_{1}\right)}\right]+l_{\mathrm{bs}}-l_{\mathrm{s}} \equiv d_{\mathrm{s}},
$$

where $\rho \equiv r_{\mathrm{s}}^{\prime} / r_{\mathrm{bs}}$.

When the beamstop criterion dominates a quadratic inequality in $\lambda_{2}$ is reached:

$$
\begin{aligned}
& \lambda_{2}^{2}\left(4 \rho \lambda_{0}+2 \rho \lambda_{0}^{2}\right)+\lambda_{2}\left(6 \rho \lambda_{0}+4 \rho \lambda_{0}^{2}-\lambda_{0}-2 \lambda_{1}-2 \lambda_{0} \lambda_{1}\right) \\
& \quad+\left[2 \rho \lambda_{0}\left(1+\lambda_{0}\right)-\lambda_{0}-\lambda_{1}-2 \lambda_{0} \lambda_{1}\right] \geq 0 .
\end{aligned}
$$

The left-hand side has one positive and one negative root in all physically relevant cases. The positive root yields a lower bound on the beamstop-limited case in terms of $d$ :
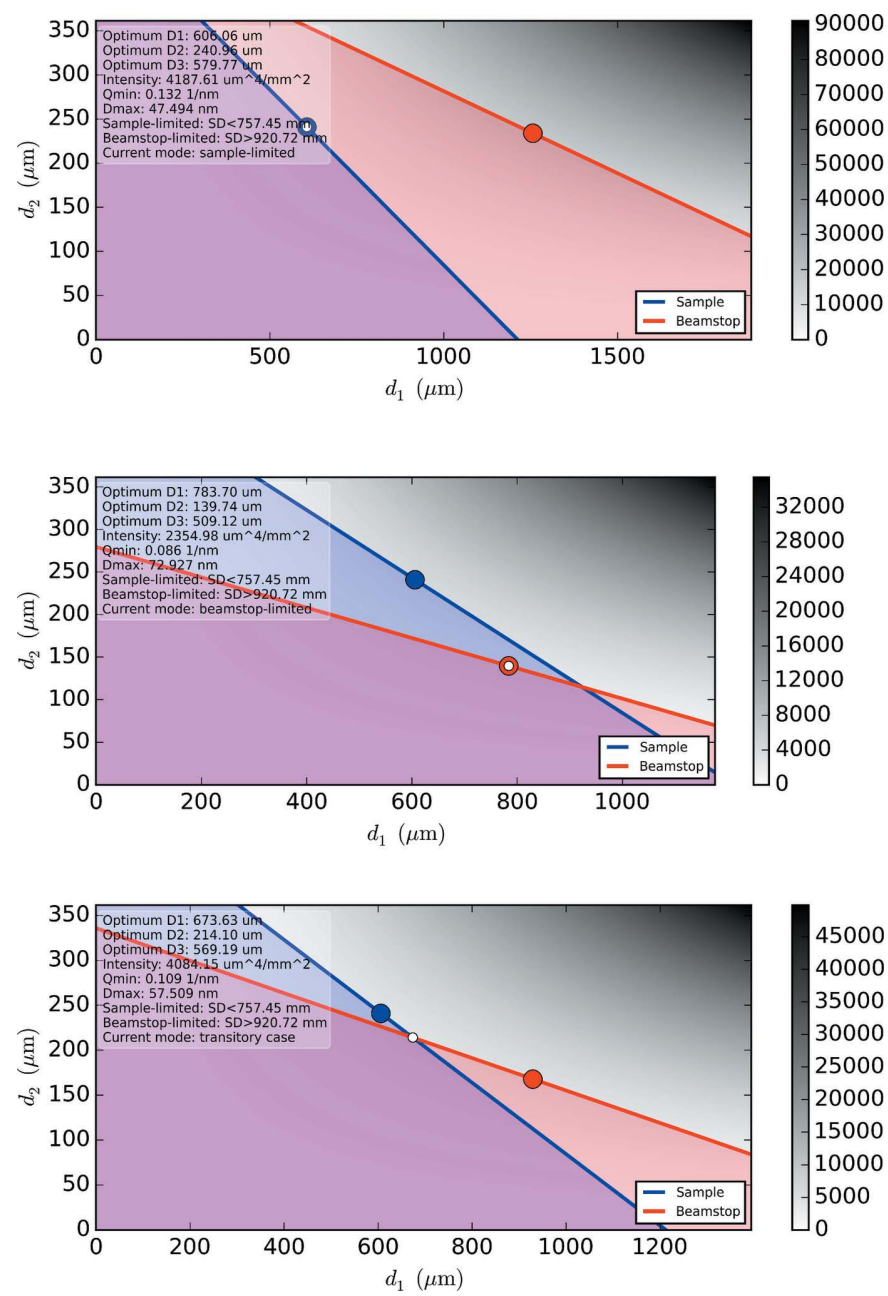

Figure 3

Graphical representations of the beam size criteria in the $d_{1}-d_{2}$ configuration space: sample-limited (top), beamstop-limited (centre) and intermediate (bottom) cases. Large solid circles mark the optimal configurations satisfying either of the constraints. The white dot marks the optimum setup. The corresponding sample-to-detector distances are 520,800 and $1000 \mathrm{~mm}$, respectively. The remaining parameters are $l_{1}=500 \mathrm{~mm}, l_{2}=200 \mathrm{~mm}, l_{\mathrm{s}}=130 \mathrm{~mm}, l_{\mathrm{bs}}=54 \mathrm{~mm}, \quad r_{\mathrm{s}}^{\prime}=0.4 \mathrm{~mm}$, $r_{\mathrm{bs}}=2 \mathrm{~mm}, \lambda=0.15418 \mathrm{~nm}$. The greyscale background represents $\mathcal{I}$. 


$$
\begin{aligned}
d_{+, \mathrm{bs}}= & l_{2}\left(\left[\lambda_{0}+2 \lambda_{1}+2 \lambda_{0} \lambda_{1}-2 \rho \lambda_{0}\left(3+2 \lambda_{0}\right)\right]\right. \\
& \left.+\left\{4 \rho \lambda_{0}\left[\lambda_{0}(\rho+1)-2 \lambda_{1}\right]+\left(\lambda_{0}+2 \lambda_{1}+2 \lambda_{0} \lambda_{1}\right)^{2}\right\}^{1 / 2}\right) \\
& /\left[4 \rho \lambda_{0}\left(2+\lambda_{0}\right)\right]+l_{\mathrm{bs}}-l_{\mathrm{s}} .
\end{aligned}
$$

The existence of a finite $] d_{\mathrm{s}}, d_{+, \mathrm{bs}}[$, in which neither of the two criteria implies the other (Fig. 3, bottom), can be proven algebraically. In this range the optimum apertures are given by the intersection of the two lines defined by equations (5) and (6):

$$
\begin{gathered}
r_{1, \text { intermed }}=\frac{r_{\mathrm{s}}^{\prime}\left[\left(1+\lambda_{0}\right)\left(1+\lambda_{2}\right)+\lambda_{2}\right]-r_{\mathrm{bs}}\left(1+\lambda_{1}\right)}{\left(\lambda_{1}-\lambda_{0}\right)\left(1+\lambda_{2}\right)+\lambda_{1} \lambda_{2}}, \\
r_{2, \text { intermed }}=\frac{r_{\mathrm{bs}} \lambda_{1}-r_{\mathrm{s}}^{\prime} \lambda_{0}\left(1+\lambda_{2}\right)}{\left(\lambda_{1}-\lambda_{0}\right)\left(1+\lambda_{2}\right)+\lambda_{1} \lambda_{2}} .
\end{gathered}
$$

The main difference between the above three cases lies in how much the limits on the sample and beamstop size are exhausted. In the sample-limited geometry the whole available cross section of the sample is irradiated, making the scattering signal stronger and the results more representative. The maximum size of the beamstop is not reached, though, which means that its diameter can be reduced (or conversely the sample-to-detector distance can be increased), leading to a lower $q_{\min }$. As seen in Fig. 4, $\mathcal{I}$ is constant in the samplelimited range and starts to decrease just as the beamstop criterion begins not to be satisfied by the sample constraint.

After the intermediate range the beamstop-limited case ensues, in which the beam size at the sample does not reach its upper limit, making the camera flux low. The real solution here would be to use different pinhole-to-pinhole distances, which would make the sample-to-detector distance fall once again into the sample-limited range.

\section{Fixed apertures}

Another class of SAS instruments operates with fixed-size apertures. It is thus worth considering the dependence of the

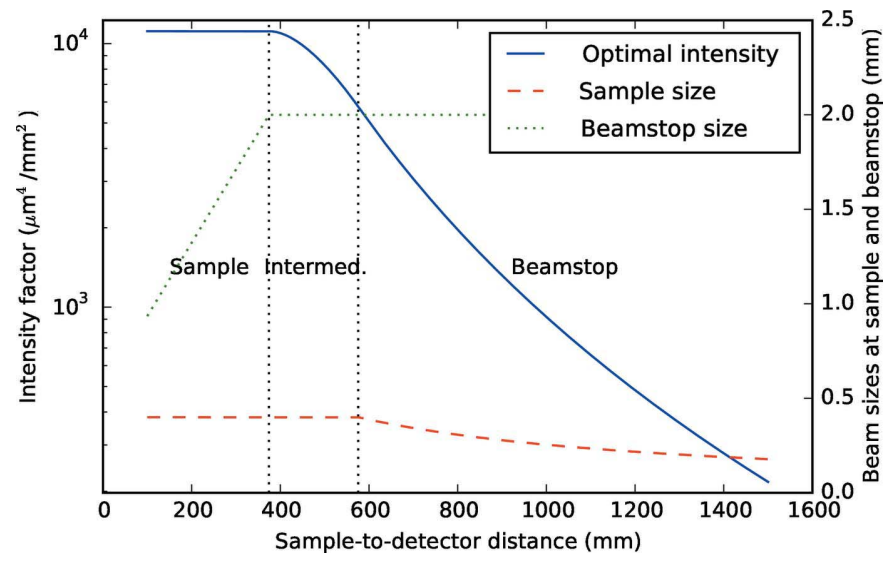

Figure 4

Maximum intensity as a function of the sample-to-detector distance. The geometrical parameters are $l_{1}=500 \mathrm{~mm}, l_{2}=100 \mathrm{~mm}, l_{\mathrm{s}}=130 \mathrm{~mm}$, $l_{\mathrm{bs}}=54 \mathrm{~mm}, r_{\mathrm{s}}^{\prime}=0.4 \mathrm{~mm}, r_{\mathrm{bs}}=2 \mathrm{~mm}$. Dotted vertical lines separate the three regions defined by the relation of criteria. collimation geometry on $l_{1}$ and $l_{2}$. Equations (2) and (3) can be reformulated as

$$
l_{1} \geq \frac{\left(r_{1}+r_{2}\right)\left(l_{2}+l_{\mathrm{s}}\right)}{r_{\mathrm{s}}^{\prime}-r_{2}}
$$

for the sample, and

$$
l_{1} \geq \frac{\left(r_{1}+r_{2}\right)\left(l_{2}+l_{3}^{\prime}\right)}{r_{\mathrm{bs}}-r_{2}\left(1+2 l_{3}^{\prime} / l_{2}\right)}
$$

for the beamstop, where $l_{3}^{\prime} \equiv l_{3}-l_{\text {bs }}$. Both inequalities give lower bounds for $l_{1}$ in terms of $l_{2}$, as represented graphically in Fig. 5.

Because $r_{1}$ and $r_{2}$ are fixed, $\mathcal{I}$ can be maximized by minimizing $l_{1}$. Since $l_{2}$ is a free parameter entering both inequalities above, a minimum $l_{1}$ can be found, satisfying the two lower bounds simultaneously. The corresponding $l_{2}$ is obtained as the positive root of the following second-order polynomial:

$$
l_{2}^{2}\left(r_{\mathrm{bs}}-r_{\mathrm{s}}^{\prime}\right)-l_{2}\left(l_{3}^{\prime} r_{2}-l_{\mathrm{s}} r_{\mathrm{bs}}+l_{\mathrm{s}} r_{2}+l_{3}^{\prime} r_{\mathrm{s}}^{\prime}\right)-2 l_{\mathrm{s}} l_{3}^{\prime} r_{2}=0 .
$$

The value for $l_{1}$ is then obtained by substituting the resulting $l_{2}$ into, for example, equation (14).

\section{Global optimization}

In special cases, such as when constructing a new instrument, all four of $l_{1}, l_{2}, r_{1}$ and $r_{2}$ are free for continuous variation. Apart from the sample and beamstop size, further limitations may also arise, including the highest and lowest allowed aperture sizes, the maximum camera length etc. Finding an analytical solution for the optimum geometry is feasible in this case only by a Monte Carlo approach after fixing $l_{\mathrm{s}}, l_{\mathrm{bs}}$ and $d$. The optimal geometry (maximizing $\mathcal{I}$ while satisfying all the criteria) is then found in terms of the four parameters.

The optimization routine starts with a random state conforming to the criteria. Next, a small modification is done in one of the parameters. If this results in the violation of any of the constraints, the change is rejected and a new trial ensues. If all of the criteria are met, the change is accepted if the difference in $\mathcal{I}$ is positive, or rejected with a probability of $1-\exp (\Delta \mathcal{I} / T)$ if it is negative. Here $T$ is the equivalent of the

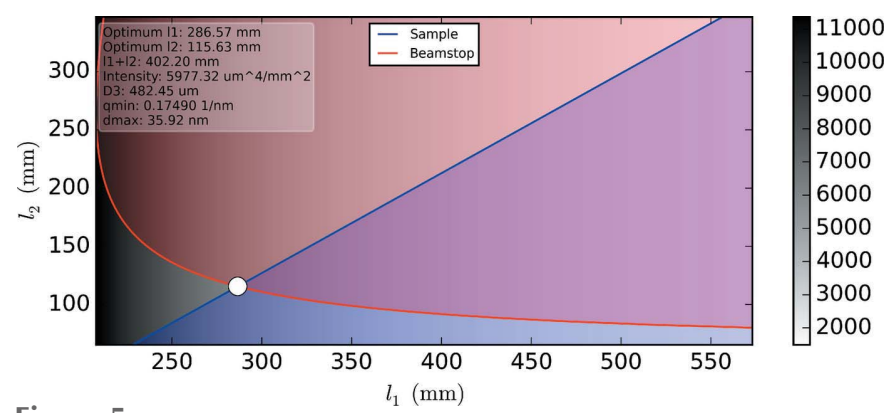

Figure 5

Geometrical representation of the sample and beamstop size criteria in the $l_{1}-l_{2}$ space. The diameters of the first and second apertures are 500 and $200 \mu \mathrm{m}$, respectively. $l_{\mathrm{s}}=130 \mathrm{~mm}, l_{\mathrm{bs}}=54 \mathrm{~mm}, \quad r_{\mathrm{s}}^{\prime}=0.4 \mathrm{~mm}$, $r_{\mathrm{bs}}=2 \mathrm{~mm}, d=520 \mathrm{~mm}$. The shaded areas signify the fulfilment of the respective criteria. 
Boltzmann energy scale, governing the size of the fluctuations in $\mathcal{I}$.

Several runs of this loop are executed, each starting where the previous one left off. At the end of each run the acceptance ratio is evaluated, and $T$ is tuned to ensure that the ratio remains near a given value, e.g. 0.4. The optimum setup is always kept and is given as a result.

\section{Discrete variations in the apertures and the separations}

In CREDO (Wacha et al., 2014) a different approach had to be implemented, as all the apertures and their spacings can be varied, but only in discrete steps. Calculating the optimum collimation for a given problem therefore involves a bruteforce filtering of all the possible configurations - that is, all combinations of available pinholes and spacing elements against an acceptance interval for $r_{\mathrm{s}}^{\prime}$ and $r_{\mathrm{bs}}$. The accepted configurations are then sorted according to $\mathcal{I}$.

\section{Software for an optimum collimation}

The above described results have been implemented in SASCollOpt.py (see the supporting information), a proof-ofconcept software implemented in Python (version 3), using the freely available packages numpy (Oliphant, 2007) and matplotlib (Hunter, 2007) for numerical operations and plotting, respectively, and the native $T k$ widget set. More details can be found in the supporting information.

\section{Examples}

In CREDO, water-based samples are measured in approximately $1 \mathrm{~mm}$-thick borosilicate capillaries. This limits the maximum beam size at the sample position to 0.8 or $0.9 \mathrm{~mm}$. Cylindrical beamstops are currently available in 4 and $2.6 \mathrm{~mm}$ diameters. The typical sample-to-detector distance for the $q$ range of $[0.2,5] \mathrm{nm}^{-1}$ is approximately $520 \mathrm{~mm}$. Before the development of SASCollOpt.py, a naïve setup was used with pinholes of diameters 500, 400 and $600 \mu \mathrm{m}$ and 816 and $312 \mathrm{~mm}$ spacings. According to equation (4) $\mathcal{I} \simeq$ $2949 \mu \mathrm{m}^{4} \mathrm{~mm}^{-2}$, which corresponds to $\sim 150000$ arbitrary units of measured intensity (inverse of the absolute intensity scaling factor). Nowadays we use a similar setup with pinholes of 600,400 and $750 \mu \mathrm{m}$ (spacings 612 and $212 \mathrm{~mm}$ ), with $\mathcal{I} \simeq 7549 \mu \mathrm{m}^{4} \mathrm{~mm}^{-2}(2.5 \times$ increase $)$, while the measured relative intensity is nearly $224000(1.5 \times$ the original value $)$. While the improvement is substantial, it is less than expected, which may be due to the different alignment of the optics of the X-ray source between the two setups.

Monte Carlo simulations revealed that the maximization of $r_{1}$ - even at the cost of lengthening $l_{1}$ - always increases the available intensity at the sample, consistent with the studies of Bolduan \& Bear (1949) and Pedersen (2004). Consequently, using the widest available pinhole in the first position (1250 $\mu \mathrm{m}$, the others being 500 and $750 \mu \mathrm{m}$ and with spacings 1520 and $212 \mathrm{~mm}), \mathcal{I}$ became nearly $8300 \mu \mathrm{m}^{4} \mathrm{~mm}^{-2}$, while the measured intensity increased to 264000 . The increase is still substantial, although once again non-proportional, because the size of the first aperture is near the cross section of the raw beam of the X-ray source $(1.1 \times 1.5 \mu \mathrm{m})$; thus the approximation leading to equation (4) starts to break down.

Another general property of the structures created by the Monte Carlo algorithm is that the size of the second aperture is smaller than that of the first one, a feature already reported by Bolduan \& Bear (1949).

The instrumental background is also a crucial point. In a typical setup for weak scatterers on CREDO the basic parameters are $l_{1}=1116 \mathrm{~mm}, l_{2}=212 \mathrm{~mm}, r_{1}=625 \mu \mathrm{m}, r_{2}=$ $300 \mu \mathrm{m}$. The calculated radius of the anti-scatter pinhole is $r_{3}=297 \mu \mathrm{m}$; thus the next available size, i.e. $300 \mu \mathrm{m}$, is employed. A $4 \mathrm{~mm}$ beamstop is used with a $523.7 \mathrm{~mm}$ sampleto-detector distance, yielding a $q$ range from 0.2 to $5.33 \mathrm{~nm}^{-1}$. An empty beam measurement for more than $30 \mathrm{~min}$ revealed the instrumental background (including effects from cosmic radiation) to be $1.7 \times 10^{-6}$ counts $\mathrm{s}^{-1}$ pixel $^{-1}$, corresponding to less than 0.5 counts $\mathrm{s}^{-1}$ on the whole detector surface. Measurements of cosmic and natural background radiation (with the X-ray generator turned off) yielded the same result. By comparison, scattering of water with this setup is nearly 3 counts $\mathrm{s}^{-1}$ on the whole surface after removing most of the traces of high-energy particles from the detector images (dezingering).

\section{Conclusion}

Alignment and fine-tuning of a three-aperture collimating system is basically a straightforward procedure: with a little practice, good configurations can be easily achieved with relatively high intensity and reasonably low instrumental background. In contrast to high-brilliance synchrotron beamlines (e.g. Urban et al., 2003; Nielsen et al., 2012; Blanchet et al., 2015), where obtaining results of good statistical quality is a matter of a few seconds, laboratory SAXS instruments and small-angle neutron scattering facilities suffer from very low flux; thus a compromise must be made between the signal-tonoise ratio (beam intensity) and the spatial resolution (attainable range of $q$ ), while keeping the instrumental background as low as possible.

A phase-space analysis of the three-pinhole collimation scheme, based on a simple analytical model for the intensity of the transmitted radiation, enabled the determination of the optimal setup, with constraints on the beam cross section at the sample and the beamstop.

Optimal aperture sizes have been determined for fixed pinhole spacings and continuously variable apertures. An analysis of the dependence on the sample-to-detector distance revealed that the highest intensity is obtained when only the limit on the sample size is exhausted.

The converse, although less typical, situation of continuously variable spacings has also been solved.

A general case relevant in the design of new pinhole SAXS cameras, where both the apertures and their spacings are freely variable, has been treated using a Monte Carlo method. 
It was found that, in order to maximize the intensity, the first pinhole should be as large as possible, even at the cost of a lengthened collimating system. This was also confirmed in the brute-force enumeration approach of discrete pinhole apertures and spacings.

Finally, SASCollOpt.py, a proof-of-concept program, has been developed, wherein the results of this study have been implemented. It is made available as supporting information. Up-to-date versions and bug fixes can be requested from the author.

\section{Acknowledgements}

The author is indebted to Dr Zoltán Varga and Dr Attila Bóta for helpful discussions and support. This work has been funded by the Hungarian National Scientific Research Fund (grant No. CNK 81052) and by a grant from the Central Hungarian Operative Program (KMOP-1.1.2-07/1-2008-0002).

\section{References}

Blanchet, C. E., Spilotros, A., Schwemmer, F., Graewert, M. A., Kikhney, A., Jeffries, C. M., Franke, D., Mark, D., Zengerle, R., Cipriani, F., Fiedler, S., Roessle, M. \& Svergun, D. I. (2015). J. Appl. Cryst. 48, 431-443.

Bolduan, O. E. A. \& Bear, R. S. (1949). J. Appl. Phys. 20, 983-992.

Bóta, A. (2013). J. Appl. Cryst. 46, 573-576.

Bu, Z., Perlo, A., Johnson, G. E., Olack, G., Engelman, D. M. \& Wyckoff, H. W. (1998). J. Appl. Cryst. 31, 533-543.

Fritz, G. \& Bergmann, A. (2006). J. Appl. Cryst. 39, 64-71.
Haubold, H. G., Gruenhagen, K., Wagener, M., Jungbluth, H., Heer, H., Pfeil, A., Rongen, H., Brandenberg, G., Moeller, R., Matzerath, J., Hiller, P. \& Halling, H. (1989). Rev. Sci. Instrum. 60, 1943-1946. Hendricks, R. W. (1978). J. Appl. Cryst. 11, 15-30.

Hunter, J. D. (2007). Comput. Sci. Eng. 9, 90-95.

Huxley, H. E. \& Brown, W. (1967). J. Mol. Biol. 30, 383-434.

Huxley, H. E., Brown, W. \& Holmes, K. C. (1965). Nature, 206, 1358. Jakob, H. F., Erlacher, K. \& Fratzl, P. (2003). Mater. Sci. Forum, 414415, 411-418.

Kirby, N. M., Mudie, S. T., Hawley, A. M., Cookson, D. J., Mertens, H. D. T., Cowieson, N. \& Samardzic-Boban, V. (2013). J. Appl. Cryst. 46, 1670-1680.

Knaapila, M., Svensson, C., Barauskas, J., Zackrisson, M., Nielsen, S. S., Toft, K. N., Vestergaard, B., Arleth, L., Olsson, U., Pedersen, J. S. \& Cerenius, Y. (2009). J. Synchrotron Rad. 16, 498-504.

Kratky, O. \& Stabinger, H. (1984). Colloid Polym. Sci. 262, 345-360. Lambard, J. \& Zemb, Th. (1991). J. Appl. Cryst. 24, 555-561.

Narayanan, T., Diat, O. \& Bösecke, P. (2001). Nucl. Instrum. Methods Phys. Res. Sect. A, 467-468, 1005-1009.

Nielsen, S. S., Møller, M. \& Gillilan, R. E. (2012). J. Appl. Cryst. 45, 213-223.

Oliphant, T. E. (2007). Comput. Sci. Eng. 9, 10-20.

Pedersen, J. S. (2004). J. Appl. Cryst. 37, 369-380.

Rehm, C., Brûlé, A., Freund, A. K. \& Kennedy, S. J. (2013). J. Appl. Cryst. 46, 1699-1704.

Schelten, J. \& Hendricks, R. W. (1975). J. Appl. Cryst. 8, 421-429.

Urban, V., Panine, P., Ponchut, C., Boesecke, P. \& Narayanan, T. (2003). J. Appl. Cryst. 36, 809-811.

Wacha, A., Varga, Z. \& Bóta, A. (2014). J. Appl. Cryst. 47, 1749-1754.

Wignall, G. D., Lin, J. S. \& Spooner, S. (1990). J. Appl. Cryst. 23, 241245.

Zemb, Th., Taché, O., Né, F. \& Spalla, O. (2003a). Rev. Sci. Instrum. 74, 2456-2462.

Zemb, T., Tache, O., Né, F. \& Spalla, O. (2003b). J. Appl. Cryst. 36, 800-805. 